\title{
Research on Well-Lighted Room Holographic Technology
}

\author{
Ji Hongbo ${ }^{1}$, Zhang $\mathrm{Li}^{2}$,a \\ ${ }^{1}$ School of Computer, Tonghua Normal University, Tonghua 134002, China. \\ ${ }^{2}$ School of Physics, Tonghua Normal University, Tonghua 134002, China. \\ aCorresponding author: dbcy9999@163.com
}

Keywords: well-Lighted room, holographic, diffuse, reflection hologram.

Abstract. Well-Lighted room holographic technology is to make people better understand the principle and characteristic of hologram ,master the holographic method of diffuse objects, and produce a diffuse three-dimensional hologram. To grasp the method of reflection holographic photography, learn to make the object of the white light reflection hologram and further familiar with light path adjustment method.

\section{Introduction}

With the rapid development of science and technology, people more and more high to the requirement of photographic techniques. Well-lighted room holographic technology is to make people better understand the principle and characteristic of holographic, master the holographic method of diffuse objects, diffuse three-dimensional hologram. To grasp the method of reflection holographic photography, learn to make the object of the white light reflection hologram. Further familiar with light path adjustment method.

\section{The History of the Holographic}

On the sense that 70-80 percent of the information human gets comes from visual cue the science and technology for imaging from a large part of information science .Today's imaging industry is being challenged to find more scientific and attractive ways to present the information, it will be the 3D imaging and that holographic imaging is the ultimate 3D visualization tool.

Holography 1-8 recording9 and reconstruction of the complex in the field of science with a higher frequency of light wave and electromagnetic wave field, is Dr. Dennis Gabor invented in 1948, in 1971, was awarded the Nobel Prize for physics.

In the past few years, holography has been advanced to a stage, and it can promote the development of numerous optical and optical technologies.

For example, you can use X-ray holography, the formation of bones and organs of three-dimensional images.

Holographic optical tweezers use the computer generated hologram to create arbitrary three-dimensional configurations of optical trap for capturing and mesoscopic moving objects.

In adaptive optics, the modulation optical holography in all kinds of optical distortion correction system such as the human eye or the atmosphere.

Recent research suggests that light can be strongly scattering opaque material is focused on the space shape of wavefront when sending.

\section{Reflective Holographic Principle}

Reflection hologram and transmission hologram distinction, is based on dry plate boundaries. If "o" with the reference light from dry version of the same side, the " $\mathrm{R}$ " is a transmission hologram. If "O" with the reference light from dry plate on both sides of the incident, the " $\mathrm{R}$ " is called reflection hologram. 


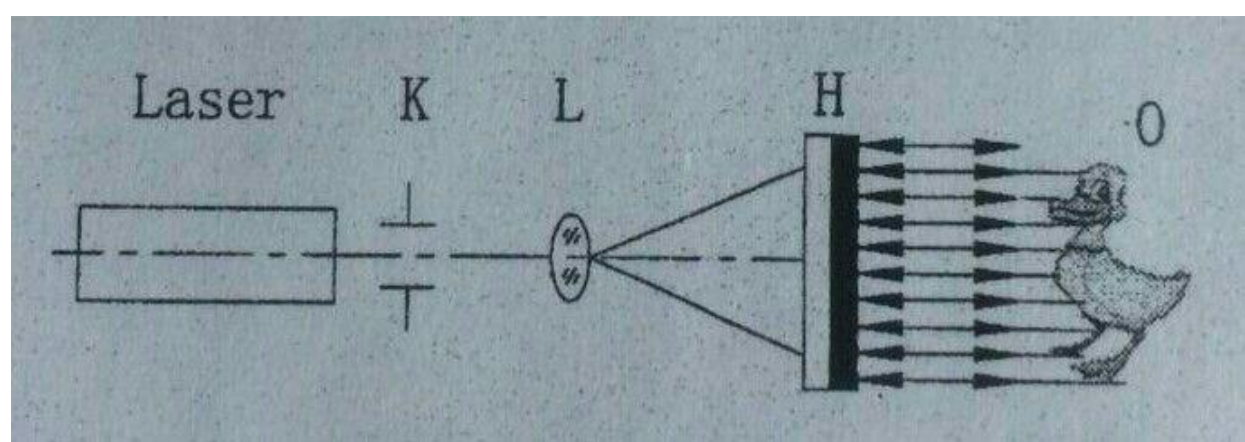

FIGURE1.Light source - He - Ne laser (or semiconductor laser), 30 mw power > K - L - beam ex pander exposure timer $\mathrm{H}$ - light polymer $\mathrm{O}$ - small objects

\section{Reflective Holographic Experiment Step}

$<1>$ Will light source, exposure timer, beam expander, dry plate, small objects into a coaxial (contour) $<2>$ Move beam expander, expanded beam diameter slightly larger than the object.(too big, light energy is lost; too small, the object according to not complete).

$<3>$ Small objects near the dry plate as far as possible, so that light intensity of light.

$<4>$ Light path lined up, check the stability, including optical sat on the screws are tight, magnetic bridge is to absorb, etc.

$<5>$ Mount dry plate, surface toward the small objects, installed, stable 2 minutes (eliminating stress) $<6>$ Exposure time according to the size of the light source power, select the appropriate exposure time on the strength of the reflective object (reference: if the light source power $30 \mathrm{mw}$, small objects is magnetic or glass, exposure time in about 20-30 seconds).

Exposure, it is strictly prohibited to loud and move around, it is forbidden to touch the workbench and dynamic table.

$<7>$ After exposure, to deal with distilled water, isopropyl alcohol.

\section{Processing}

$<1>$ The dry plate after exposure, after take off, put in distilled water for 15 to 30 seconds, after th e exposure of molecular fully absorbing water, completely dissolved excess reagent in the dry plate, to attain to the maximum refractive index modulation.

$<2>$ Out into the concentration of $40 \%$ isopropanol dehydration in 1 minute;

$<3>$ Out into the concentration of $60 \%$ isopropanol dehydration in 1 minute;

$<4>$ Out into the concentration of $80 \%$ isopropanol dehydration 15 seconds;

$<5>$ Out into the concentration of 100\% isopropanol dehydration 60-80 seconds, in the image clear, bright, color is pale red or yellow.

$<6>$ Out quickly; with hot hair dryer will dry plate quickly dry, until the hologram is golden brown, the clear and bright.(The reflection hologram)

$<7>$ For general hologram, is not a package.

But if you want to permanently kept, also need packaging according to the following methods: with a same size with the hologram size piece of glass, clean, dry, which is covering the hologram latex surface, sealed with sealant, after the room temperature curing, which is a permanent record of the hologram.(Note: the sealant can be used in Tianjin production of HY - 914 quick-drying glue.) 


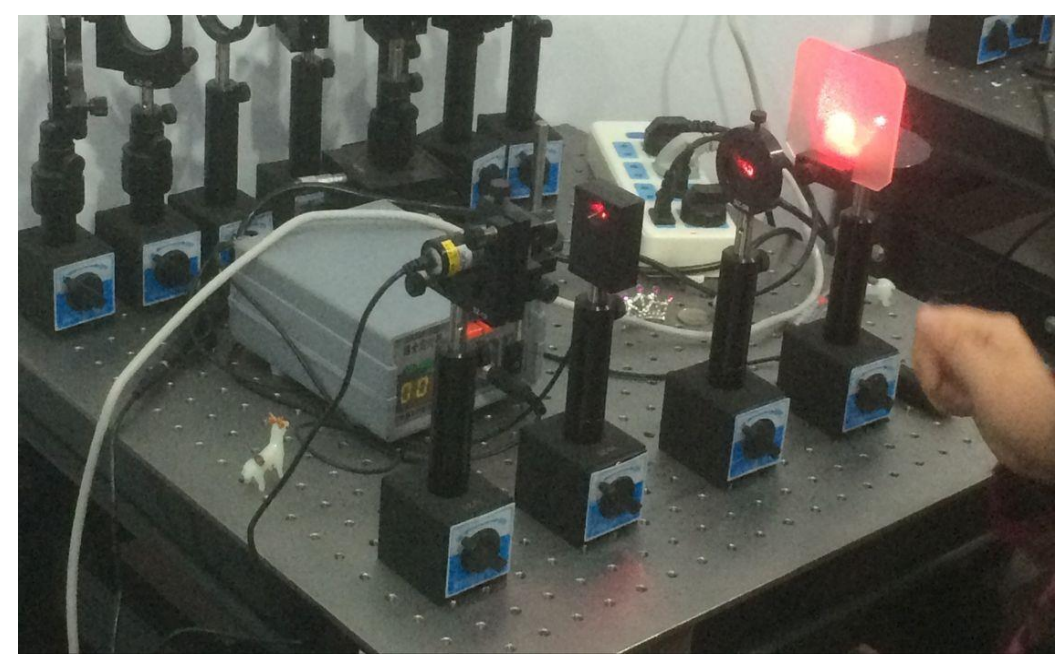

FIGURE2. The experimental principle diagram

\section{The Photography Works Display}

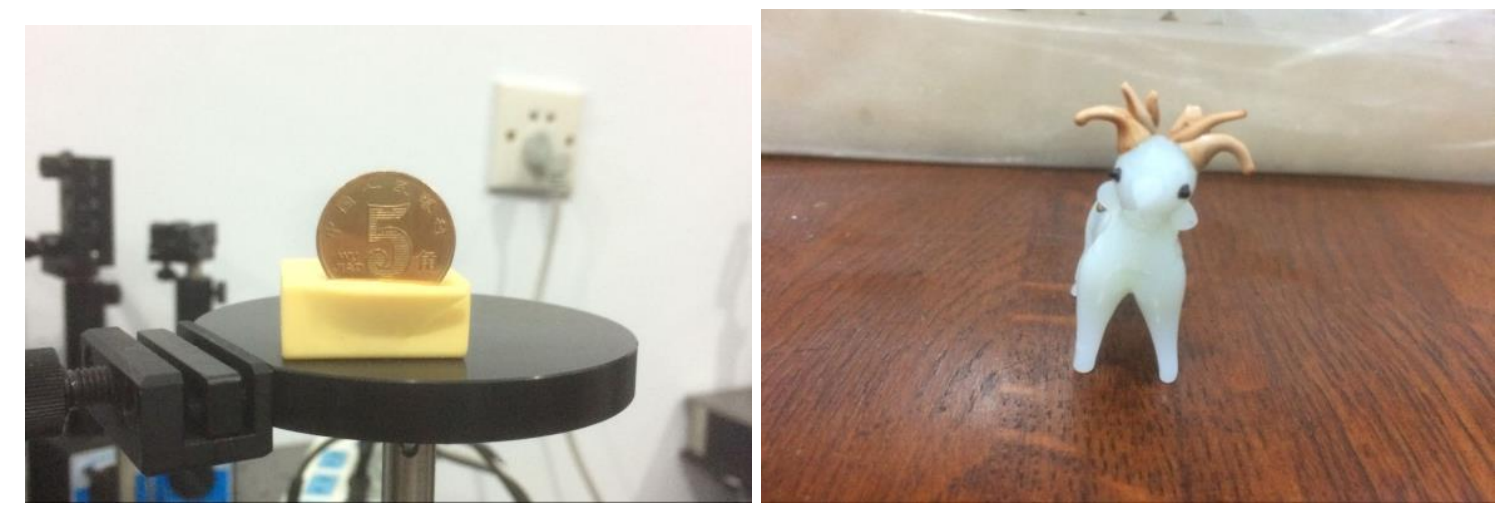

\section{FIGURE3. Real figure}

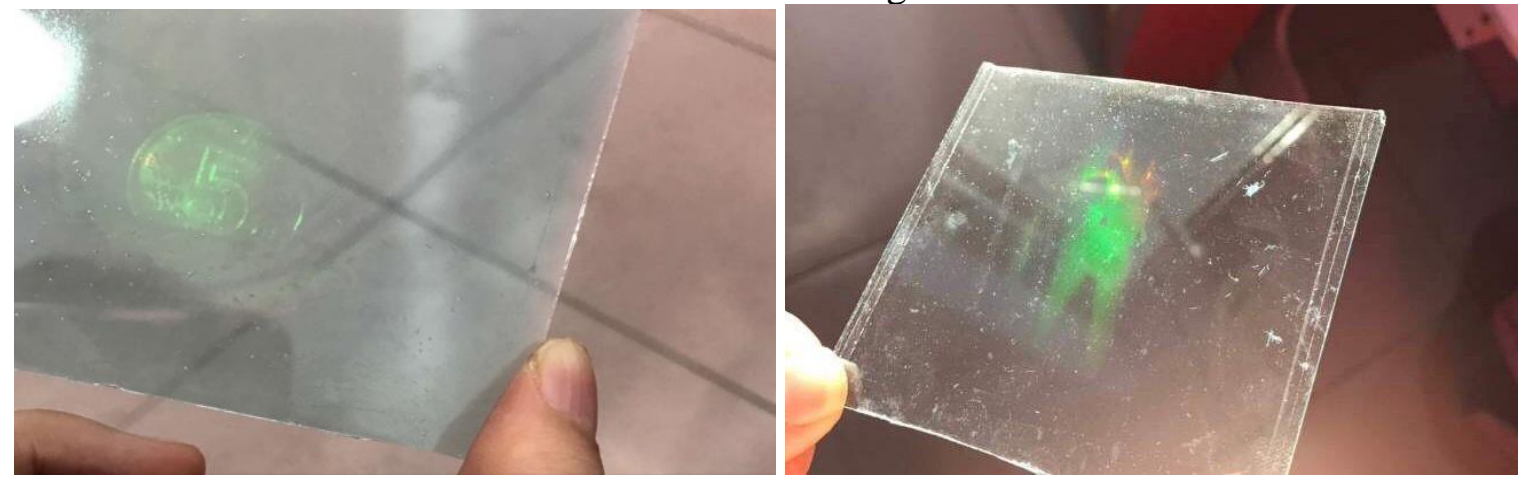

FIGURE4. Photographic renderings

\section{Well-lighted Room Holographic Advantages}

One, because the photography under the condition of a light, good light path adjustment

Two, operation steps, playability strong under the dim room.

Three, the cut piece can be operated in the room, and concise

Four, when the flush, only a ratio of reagents isopropyl alcohol, easy to operate.

Five, can see objects in light of the three dimensional images, don't need a red glow.

\section{Conclusion}

The holographic technology 10 is a kind of record for the object of reflection wave amplitude and e qual to all the information of the new type of photography. 
Ordinary camera is recording intensity distribution on the surface of the object, it cannot record the phase of the reflected light, thus losesthe stereo feeling.Hologram using laser as light source, the light of light source is divided into two beams, a bunch of directly toward the plates and the other a bunch of shooting again after the reflection of the subject of plates.Two beams of light interfere, superimposed on the plates of each point on the plate photosensitive degree not only with strength also varies according to the relations between the two phases of beam of light.So hologram not only recorded the reflective intensity of objects, also recorded the phase information.Directly to the human eye to see this kind of plate, can only see like fingerprints of interference fringes, but if use laser to irradiate it, the human eye can see through the negatives original photographed the same $3 \mathrm{~d}$ as object.

\section{Acknowledgments}

This article thanks Tonghua Normal University youth corps committee college students entrepreneu rial projects "Research on Well-Lighted Room Holographic Technology."

\section{References}

[1] Hao Zhang, Lighting effects rendering in three-dimensional computer-generated holographic display, Elsevier journal 10.1016/j.optcom.2016.03.013.

[2] Hengyan Zhou,Method to fabricate orthogonal crossed gratings based on a dual Lloyd's mirror interferometer, Elsevier journal 10.1016/j.optcom.2015.10.017(Holographic interferometry).

[3] Ángel G. Augier, Geometric Plane Shapes for Computer-Generated Holographic Engraving Codes, Elsevier journal 10.1016/j.optcom.2016.11.018.

[4] Ján Vavro, The Optimisation of the Materials Properties for the Passenger Cars in Dependence on Defect Distribution at the Dynamic Loading, Elsevier journal 10.1016/j.proeng.2016.01.183.

[5] Yoshiharu Morimoto, Image Processing for Optical Methods to Analyze Shape, Deformation, Stress and Strain, Trans Tech magazine 10.4028/www.scientific.net/KEM.326-328.13.

[6] Wen-Bo Cao, Binary hologram generation based on discrete wavelet transform, Elsevier journal 10.1016/j.ijleo.2015.10.115.

[7] Oleg T. Kamenev, An adaptive fibre-optic interferometer with low cutoff frequency, Elsevier journal 10.1016/j.psra.2015.11.008.

[8] M. Lesaffre, ACOUSTO-OPTIC IMAGING TECHNIQUES FOR OPTICAL DIAGNOSIS, Elsevier journal 10.3182/20060920-3-FR-2912.00007.

[9] A.M. Nastas, Influence of corona discharge on holographic recording of diffraction gratings in $\mathrm{Cu}$-chalcogenide film structures, Elsevier journa10.1016/j.jnoncrysol.2016.02.004.

[10]L. B. Lesem, Scientific Applications: Computer synthesis of Holograms for 3-D display, Journal of the American association of computer,1968-10,10.1145/364096.364111 